Analele Universităţii de Vest, Timişoara

Seria Matematică - Informatică

LV , 1, (2017), 63-86

\title{
Hermitian Forms and Inequalities for Sequences and Power Series of Operators in Hilbert Spaces
}

\author{
S. S. Dragomir ${ }^{1,2}$
}

\begin{abstract}
By the use of some inequalities for nonnegative Hermitian forms various inequalities for sequences and power series of bounded linear operators in complex Hilbert spaces are established. Applications for some fundamental functions of interest are also given.
\end{abstract}

AMS Subject Classification (2000). 47A63; 47A99.

Keywords. Hermitian forms, Hilbert spaces, Bounded linear operators, Normal operators, Operator norm and numerical radius

\section{Introduction}

Let $\mathbb{K}$ be the field of real or complex numbers, i.e., $\mathbb{K}=\mathbb{R}$ or $\mathbb{C}$ and $X$ be a linear space over $\mathbb{K}$.

Definition 1. A functional $(\cdot, \cdot): X \times X \rightarrow \mathbb{K}$ is said to be a Hermitian form on $X$ if

(H1) $(a x+b y, z)=a(x, z)+b(y, z)$ for $a, b \in \mathbb{K}$ and $x, y, z \in X$;

(H2) $(x, y)=\overline{(y, x)}$ for all $x, y \in X$. 
The functional $(\cdot, \cdot)$ is said to be positive semi-definite on a subspace $Y$ of $X$ if

(H3) $(y, y) \geq 0$ for every $y \in Y$,

and positive definite on $Y$ if it is positive semi-definite on $Y$ and

(H4) $(y, y)=0, y \in Y$ implies $y=0$.

The functional $(\cdot, \cdot)$ is said to be definite on $Y$ provided that either $(\cdot, \cdot)$ or $-(\cdot, \cdot)$ is positive semi-definite on $Y$.

When a Hermitian functional $(\cdot, \cdot)$ is positive-definite on the whole space $X$, then, as usual, we will call it an inner product on $X$ and will denote it by $\langle\cdot, \cdot\rangle$.

We use the following notations related to a given Hermitian form $(\cdot, \cdot)$ on $X$ :

$$
X_{0}:=\{x \in X \mid(x, x)=0\}, K:=\{x \in X \mid(x, x)<0\}
$$

and, for a given $z \in X$,

$$
X^{(z)}:=\{x \in X \mid(x, z)=0\} \quad \text { and } \quad L(z):=\{a z \mid a \in \mathbb{K}\} .
$$

The following fundamental facts concerning Hermitian forms hold:

Theorem 1 (Kurepa, $1968[27])$. Let $X$ and $(\cdot, \cdot)$ be as above.

1. If $e \in X$ is such that $(e, e) \neq 0$, then we have the decomposition

$$
X=L(e) \bigoplus X^{(e)}
$$

where $\bigoplus$ denotes the direct sum of the linear subspaces $X^{(e)}$ and $L(e)$;

2. If the functional $(\cdot, \cdot)$ is positive semi-definite on $X^{(e)}$ for at least one $e \in K$, then $(\cdot, \cdot)$ is positive semi-definite on $X^{(f)}$ for each $f \in K$;

3. The functional $(\cdot, \cdot)$ is positive semi-definite on $X^{(e)}$ with $e \in K$ if and only if the inequality

$$
|(x, y)|^{2} \geq(x, x)(y, y)
$$

holds for all $x \in K$ and all $y \in X$;

4. The functional $(\cdot, \cdot)$ is semi-definite on $X$ if and only if the Schwarz's inequality

$$
|(x, y)|^{2} \leq(x, x)(y, y)
$$

holds for all $x, y \in X$ 
5. The case of equality holds in (1.3) for $x, y \in X$ and in (1.2), for $x \in K$, $y \in X$, respectively; if and only if there exists a scalar $a \in \mathbb{K}$ such that

$$
y-a x \in X_{0}^{(x)}:=X_{0} \cap X^{(x)} .
$$

Let $X$ be a linear space over the real or complex number field $\mathbb{K}$ and let us denote by $\mathcal{H}(X)$ the class of all positive semi-definite Hermitian forms on $X$, or, for simplicity, nonnegative forms on $X$.

If $(\cdot, \cdot) \in \mathcal{H}(X)$, then the functional $\|\cdot\|=(\cdot, \cdot)^{\frac{1}{2}}$ is a semi-norm on $X$ and the following equivalent versions of Schwarz's inequality hold:

$$
\|x\|^{2}\|y\|^{2} \geq|(x, y)|^{2} \quad \text { or } \quad\|x\|\|y\| \geq|(x, y)|
$$

for any $x, y \in X$.

Now, let us observe that $\mathcal{H}(X)$ is a convex cone in the linear space of all mappings defined on $X^{2}$ with values in $\mathbb{K}$, i.e.,

(e) $(\cdot, \cdot)_{1},(\cdot, \cdot)_{2} \in \mathcal{H}(X)$ implies that $(\cdot, \cdot)_{1}+(\cdot, \cdot)_{2} \in \mathcal{H}(X)$;

(ee) $\alpha \geq 0$ and $(\cdot, \cdot) \in \mathcal{H}(X)$ implies that $\alpha(\cdot, \cdot) \in \mathcal{H}(X)$.

We can introduce on $\mathcal{H}(X)$ the following binary relation [22]:

$$
(\cdot, \cdot)_{2} \geq(\cdot, \cdot)_{1} \quad \text { if and only if } \quad\|x\|_{2} \geq\|x\|_{1} \quad \text { for all } x \in X .
$$

We observe that the following properties hold:

(b) $(\cdot, \cdot)_{2} \geq(\cdot, \cdot)_{1}$ for all $(\cdot, \cdot) \in \mathcal{H}(X)$;

(bb) $(\cdot, \cdot)_{3} \geq(\cdot, \cdot)_{2}$ and $(\cdot, \cdot)_{2} \geq(\cdot, \cdot)_{1}$ implies that $(\cdot, \cdot)_{3} \geq(\cdot, \cdot)_{1}$;

(bbb) $(\cdot, \cdot)_{2} \geq(\cdot, \cdot)_{1}$ and $(\cdot, \cdot)_{1} \geq(\cdot, \cdot)_{2}$ implies that $(\cdot, \cdot)_{2}=(\cdot, \cdot)_{1}$;

i.e., the binary relation defined by (1.5) is an order relation on $\mathcal{H}(X)$.

While (b) and (bb) are obvious from the definition, we should remark, for (bbb), that if $(\cdot, \cdot)_{2} \geq(\cdot, \cdot)_{1}$ and $(\cdot, \cdot)_{1} \geq(\cdot, \cdot)_{2}$, then obviously $\|x\|_{2}=\|x\|_{1}$ for all $x \in X$, which implies, by the following well known identity:

$$
(x, y)_{k}:=\frac{1}{4}\left[\|x+y\|_{k}^{2}-\|x-y\|_{k}^{2}+i\left(\|x+i y\|_{k}^{2}-\|x-i y\|_{k}^{2}\right)\right]
$$

with $x, y \in X$ and $k \in\{1,2\}$, that $(x, y)_{2}=(x, y)_{1}$ for all $x, y \in X$. 


\section{Superadditivity and Monotonicity of Some Mappings}

Let us consider the following mapping [22]:

$$
\sigma: \mathcal{H}(X) \times X^{2} \rightarrow \mathbb{R}_{+}, \quad \sigma((\cdot, \cdot) ; x, y):=\|x\|\|y\|-|(x, y)|,
$$

which is closely related to Schwarz's inequality (1.4).

The following simple properties of $\sigma$ are obvious:

(s) $\sigma(\alpha(\cdot, \cdot) ; x, y)=\alpha \sigma((\cdot, \cdot) ; x, y)$;

(ss) $\sigma((\cdot, \cdot) ; y, x)=\sigma((\cdot, \cdot) ; x, y)$;

(sss) $\sigma((\cdot, \cdot) ; x, y) \geq 0$ (Schwarz's inequality);

for any $\alpha \geq 0,(\cdot, \cdot) \in \mathcal{H}(X)$ and $x, y \in X$.

The following result concerning the functional properties of $\sigma$ as a function depending on the nonnegative Hermitian form $(\cdot, \cdot)$ has been obtained in [22]:

Theorem 2 (Dragomir \& Mond, 1994 [22]). The mapping $\sigma$ satisfies the following statements:

(i) For every $(\cdot, \cdot)_{i} \in \mathcal{H}(X)(i=1,2)$ one has the inequality

$$
\sigma\left((\cdot, \cdot)_{1}+(\cdot, \cdot)_{2} ; x, y\right) \geq \sigma\left((\cdot, \cdot)_{1} ; x, y\right)+\sigma\left((\cdot, \cdot)_{2} ; x, y\right)(\geq 0)
$$

for all $x, y \in X$, i.e., the mapping $\sigma(\cdot ; x, y)$ is superadditive on $\mathcal{H}(X)$;

(ii) For every $(\cdot, \cdot)_{i} \in \mathcal{H}(X)(i=1,2)$ with $(\cdot, \cdot)_{2} \geq(\cdot, \cdot)_{1}$ one has

$$
\sigma\left((\cdot, \cdot)_{2} ; x, y\right) \geq \sigma\left((\cdot, \cdot)_{1} ; x, y\right)(\geq 0)
$$

for all $x, y \in X$, i.e., the mapping $\sigma(\cdot ; x, y)$ is nondecreasing on $\mathcal{H}(X)$.

Remark 1. If we consider the related mapping [22]

$$
\sigma_{r}((\cdot, \cdot) ; x, y):=\|x\|\|y\|-\operatorname{Re}(x, y)
$$

then we can show, as above, that $\sigma(\cdot ; x, y)$ is superadditive and nondecreasing on $\mathcal{H}(X)$.

Moreover, if we introduce another mapping, namely, [22]

$$
\tau: \mathcal{H}(X) \times X^{2} \rightarrow \mathbb{R}_{+}, \quad \tau((\cdot, \cdot) ; x, y):=(\|x\|+\|y\|)^{2}-\|x+y\|^{2},
$$


which is connected with the triangle inequality

$$
\|x+y\| \leq\|x\|+\|y\| \quad \text { for any } \quad x, y \in X
$$

then we observe that

$$
\tau((\cdot, \cdot) ; x, y)=2 \sigma_{r}((\cdot, \cdot) ; x, y)
$$

for all $(\cdot, \cdot) \in \mathcal{H}(X)$ and $x, y \in X$, therefore $\sigma(\cdot ; x, y)$ is in its turn a superadditive and nondecreasing functional on $\mathcal{H}(X)$.

Now consider another mapping naturally associated to Schwarz's inequality, namely [22]

$$
\delta: \mathcal{H}(X) \times X^{2} \rightarrow \mathbb{R}_{+}, \quad \delta((\cdot, \cdot) ; x, y):=\|x\|^{2}\|y\|^{2}-|(x, y)|^{2} .
$$

It is obvious that the following properties are valid:

(i) $\delta((\cdot, \cdot) ; x, y) \geq 0$ (Schwarz's inequality);

(ii) $\delta((\cdot, \cdot) ; x, y)=\delta((\cdot, \cdot) ; y, x)$;

(iii) $\delta(\alpha(\cdot, \cdot) ; x, y)=\alpha^{2} \delta((\cdot, \cdot) ; x, y)$

for all $x, y \in X, \alpha \geq 0$ and $(\cdot, \cdot) \in \mathcal{H}(X)$.

The following theorem incorporates some further properties of this functional [22]:

Theorem 3 (Dragomir \& Mond, 1994 [22]). With the above assumptions, we have:

(i) If $(\cdot, \cdot)_{i} \in \mathcal{H}(X)(i=1,2)$, then

$$
\begin{aligned}
& \delta\left((\cdot, \cdot)_{1}+(\cdot, \cdot)_{2} ; x, y\right)-\delta\left((\cdot, \cdot)_{1} ; x, y\right)-\delta\left((\cdot, \cdot)_{2} ; x, y\right) \\
& \geq\left(\operatorname{det}\left[\begin{array}{ll}
\|x\|_{1} & \|y\|_{1} \\
\|x\|_{2} & \|y\|_{2}
\end{array}\right]\right)^{2}(\geq 0) ;
\end{aligned}
$$

i.e., the mapping $\delta(\cdot ; x, y)$ is strong superadditive on $\mathcal{H}(X)$.

(ii) If $(\cdot, \cdot)_{i} \in \mathcal{H}(X)(i=1,2)$, with $(\cdot, \cdot)_{2} \geq(\cdot, \cdot)_{1}$, then

$$
\begin{aligned}
& \delta\left((\cdot, \cdot)_{2} ; x, y\right)-\delta\left((\cdot, \cdot)_{1} ; x, y\right) \\
& \geq\left(\operatorname{det}\left[\begin{array}{cc}
\|x\|_{1} & \|y\|_{1} \\
\left(\|x\|_{2}^{2}-\|x\|_{1}^{2}\right)^{\frac{1}{2}} & \left(\|y\|_{2}^{2}-\|y\|_{1}^{2}\right)^{\frac{1}{2}}
\end{array}\right]\right)^{2}(\geq 0) ;
\end{aligned}
$$

i.e., the mapping $\delta(\cdot ; x, y)$ is strong nondecreasing on $\mathcal{H}(X)$. 
Remark 2. If we consider the functional

$$
\delta_{r}((\cdot, \cdot) ; x, y):=\|x\|^{2}\|y\|^{2}-[\operatorname{Re}(x, y)]^{2},
$$

then we can state similar properties for it. We omit the details.

Consider the functional $\beta: \mathcal{H}(X) \times X^{2} \rightarrow \mathbb{R}[23]$ defined by

$$
\beta((\cdot, \cdot) ; x, y)=\left(\|x\|^{2}\|y\|^{2}-|(x, y)|^{2}\right)^{\frac{1}{2}} .
$$

It is obvious that $\beta((\cdot, \cdot) ; x, y)=[\delta((\cdot, \cdot) ; x, y)]^{\frac{1}{2}}$ and thus it is monotonic nondecreasing on $\mathcal{H}(X)$.

For the subclass $\mathcal{J P}(X)$, of all inner products defined on $X$, of $\mathcal{H}(X)$ and $y \neq 0$, we may define

$$
\gamma((\cdot, \cdot) ; x, y)=\frac{\|x\|^{2}\|y\|^{2}-|(x, y)|^{2}}{\|y\|^{2}}=\frac{\delta((\cdot, \cdot) ; x, y)}{\|y\|^{2}} .
$$

The following result may be stated (see also [23]):

Theorem 4 (Dragomir \& Mond, 1995 [23]). The functional $\gamma(\cdot ; x, y)$ is superadditive and monotonic nondecreasing on $\mathcal{J P}(X)$ for any $x, y \in X$ with $y \neq 0$.

Corollary 1. If $(\cdot, \cdot)_{i} \in \mathcal{J P}(X)$ with $(\cdot, \cdot)_{2} \geq(\cdot, \cdot)_{1}$ and $x, y \in X$ are such that $x, y \neq 0$, then:

$$
\begin{aligned}
& \delta\left((\cdot, \cdot)_{2} ; x, y\right) \geq \max \left\{\frac{\|y\|_{2}^{2}}{\|y\|_{1}^{2}}, \frac{\|x\|_{2}^{2}}{\|x\|_{1}^{2}}\right\} \delta\left((\cdot, \cdot)_{1} ; x, y\right) \\
&\left(\geq \delta\left((\cdot, \cdot)_{1} ; x, y\right)\right)
\end{aligned}
$$

or equivalently, [23]

$$
\begin{aligned}
& \delta\left((\cdot, \cdot)_{2} ; x, y\right)-\delta\left((\cdot, \cdot)_{1} ; x, y\right) \\
& \geq \max \left\{\frac{\|y\|_{2}^{2}-\|y\|_{1}^{2}}{\|y\|_{1}^{2}}, \frac{\|x\|_{2}^{2}-\|x\|_{1}^{2}}{\|x\|_{1}^{2}}\right\} \delta\left((\cdot, \cdot)_{1} ; x, y\right) .
\end{aligned}
$$

The following strong superadditivity property of $\delta(\cdot ; x, y)$ that is different from the one above holds [23]: 
Corollary 2 (Dragomir \& Mond, $1995[23])$. If $(\cdot, \cdot)_{i} \in \mathcal{J P}(X)$ and $x, y \in X$ with $x, y \neq 0$, then

$$
\begin{aligned}
& \delta\left((\cdot, \cdot)_{1}+(\cdot, \cdot)_{2} ; x, y\right)-\delta\left((\cdot, \cdot)_{1} ; x, y\right)-\delta\left((\cdot, \cdot)_{2} ; x, y\right) \\
& \geq \max \left\{\left(\frac{\|y\|_{2}}{\|y\|_{1}}\right)^{2} \delta\left((\cdot, \cdot)_{1} ; x, y\right)+\left(\frac{\|y\|_{1}}{\|y\|_{2}}\right)^{2} \delta\left((\cdot, \cdot)_{2} ; x, y\right)\right. \\
& \left.\left(\frac{\|x\|_{2}}{\|x\|_{1}}\right)^{2} \delta\left((\cdot, \cdot)_{1} ; x, y\right)+\left(\frac{\|x\|_{1}}{\|x\|_{2}}\right)^{2} \delta\left((\cdot, \cdot)_{2} ; x, y\right)\right\} \quad(\geq 0) .
\end{aligned}
$$

Remark 3. Obviously, all the inequalities above remain true if $(\cdot, \cdot)_{i}, i=1,2$ are nonnegative Hermitian forms for which we have $\|x\|_{i},\|y\|_{i} \neq 0$.

Finally, we may state the superadditivity result for the mapping $\beta$ (see [23]):

Theorem 5 (Dragomir \& Mond, 1995 [23]). The mapping $\beta$ defined by (2.7) is superadditive on $\mathcal{H}(X)$.

For various properties and results for nonnegative Hermitian forms, see the book [12].

\section{Vector Inequalities for $n$-Tuple of Operators}

Let $\mathbf{T}=\left(T_{1}, \ldots, T_{n}\right) \in \mathcal{B}(H) \times \ldots \times \mathcal{B}(H):=\mathcal{B}^{(n)}(H)$ be an $n$-tuple of bounded linear operators on the Hilbert space $(H ;\langle\cdot, \cdot\rangle)$ and $\mathbf{p}=\left(p_{1}, \ldots, p_{n}\right) \in$ $\mathbb{R}_{+}^{* n}$ an $n$-tuple of nonnegative weights not all of them equal to zero. For an $x \in H, x \neq 0$ we define

$$
\langle\mathbf{T}, \mathbf{V}\rangle_{\mathbf{p}, x}:=\sum_{j=1}^{n} p_{j}\left\langle T_{j} x, V_{j} x\right\rangle=\left\langle\left(\sum_{j=1}^{n} p_{j} V_{j}^{*} T_{j}\right) x, x\right\rangle
$$

where $\mathbf{T}=\left(T_{1}, \ldots, T_{n}\right), \mathbf{V}=\left(V_{1}, \ldots, V_{n}\right) \in \mathcal{B}^{(n)}(H)$.

We need the following result:

Lemma 1. For any $x \in H, x \neq 0$ and $\mathbf{p}=\left(p_{1}, \ldots, p_{n}\right) \in \mathbb{R}_{+}^{* n}$ we have that $\langle\cdot, \cdot\rangle_{\mathbf{p}, x}$ is a nonnegative Hermitian form on $\mathcal{B}^{(n)}(H)$.

Proof. We have that

$$
\langle\mathbf{T}, \mathbf{T}\rangle_{\mathbf{p}, x}=\left\langle\left(\sum_{j=1}^{n} p_{j} T_{j}^{*} T_{j}\right) x, x\right\rangle=\left\langle\left(\sum_{j=1}^{n} p_{j}\left|T_{j}\right|^{2}\right) x, x\right\rangle \geq 0,
$$


for any $\mathbf{T}=\left(T_{1}, \ldots, T_{n}\right) \in \mathcal{B}^{(n)}(H)$, where the operator modulus is defined by $|A|^{2}=A^{*} A, A \in \mathcal{B}(H)$.

The functional $\langle\cdot, \cdot\rangle_{p, x}$ is linear in the first variable and

$$
\begin{aligned}
\overline{\langle\mathbf{V}, \mathbf{T}\rangle_{\mathbf{p}, x}} & =\overline{\left\langle\left(\sum_{j=1}^{n} p_{j} T_{j}^{*} V_{j}\right) x, x\right\rangle}=\left\langle x,\left(\sum_{j=1}^{n} p_{j} T_{j}^{*} V_{j}\right) x\right\rangle \\
& =\left\langle\left(\sum_{j=1}^{n} p_{j} T_{j}^{*} V_{j}\right)^{*} x, x\right\rangle=\left\langle\left(\sum_{j=1}^{n} p_{j} V_{j}^{*} T_{j}\right) x, x\right\rangle=\langle\mathbf{T}, \mathbf{V}\rangle_{\mathbf{p}, x}
\end{aligned}
$$

for any $\mathbf{T}=\left(T_{1}, \ldots, T_{n}\right), \mathbf{V}=\left(V_{1}, \ldots, V_{n}\right) \in \mathcal{B}^{(n)}(H)$.

Remark 4. By the Schwarz inequality we have

$$
\left|\left\langle\left(\sum_{j=1}^{n} p_{j} V_{j}^{*} T_{j}\right) x, x\right\rangle\right|^{2} \leq\left\langle\left(\sum_{j=1}^{n} p_{j}\left|T_{j}\right|^{2}\right) x, x\right\rangle\left\langle\left(\sum_{j=1}^{n} p_{j}\left|V_{j}\right|^{2}\right) x, x\right\rangle
$$

while from the triangle inequality we have

$$
\begin{aligned}
& \left\langle\left(\sum_{j=1}^{n} p_{j}\left|T_{j}+V_{j}\right|^{2}\right) x, x\right\rangle^{1 / 2} \\
& \leq\left\langle\left(\sum_{j=1}^{n} p_{j}\left|T_{j}\right|^{2}\right) x, x\right\rangle^{1 / 2}+\left\langle\left(\sum_{j=1}^{n} p_{j}\left|V_{j}\right|^{2}\right) x, x\right\rangle^{1 / 2}
\end{aligned}
$$

for any $\mathbf{p}=\left(p_{1}, \ldots, p_{n}\right) \in \mathbb{R}_{+}^{* n}, \mathbf{T}=\left(T_{1}, \ldots, T_{n}\right), \mathbf{V}=\left(V_{1}, \ldots, V_{n}\right) \in \mathcal{B}^{(n)}(H)$ and $x \in H$.

For $\mathbf{T}=\left(T_{1}, \ldots, T_{n}\right), \mathbf{V}=\left(V_{1}, \ldots, V_{n}\right) \in \mathcal{B}^{(n)}(H) \backslash\{\mathbf{0}\}$ and $x \in H, x \neq 0$ we define the function of weights $\sigma(\cdot ; \mathbf{T}, \mathbf{V}, x): \mathbb{R}_{+}^{* n} \rightarrow[0, \infty)$ by

$$
\begin{aligned}
\sigma(\mathbf{p} ; \mathbf{T}, \mathbf{V}, x) & :=\langle\mathbf{T}, \mathbf{T}\rangle_{\mathbf{p}, x}^{1 / 2}\langle\mathbf{V}, \mathbf{V}\rangle_{\mathbf{p}, x}^{1 / 2}-\left|\langle\mathbf{T}, \mathbf{V}\rangle_{\mathbf{p}, x}\right| \\
& =\left\langle\left(\sum_{j=1}^{n} p_{j}\left|T_{j}\right|^{2}\right) x, x\right\rangle^{1 / 2}\left\langle\left(\sum_{j=1}^{n} p_{j}\left|V_{j}\right|^{2}\right) x, x\right\rangle^{1 / 2} \\
& -\left|\left\langle\left(\sum_{j=1}^{n} p_{j} V_{j}^{*} T_{j}\right) x, x\right\rangle\right|
\end{aligned}
$$


We can also define $\delta(\cdot ; \mathbf{T}, \mathbf{V}, x): \mathbb{R}_{+}^{* n} \rightarrow[0, \infty)$ by

$$
\begin{aligned}
\delta(\mathbf{p} ; \mathbf{T}, \mathbf{V}, x) & :=\langle\mathbf{T}, \mathbf{T}\rangle_{\mathbf{p}, x}\langle\mathbf{V}, \mathbf{V}\rangle_{\mathbf{p}, x}-\left|\langle\mathbf{T}, \mathbf{V}\rangle_{\mathbf{p}, x}\right|^{2} \\
& =\left\langle\left(\sum_{j=1}^{n} p_{j}\left|T_{j}\right|^{2}\right) x, x\right\rangle\left\langle\left(\sum_{j=1}^{n} p_{j}\left|V_{j}\right|^{2}\right) x, x\right\rangle \\
& -\left|\left\langle\left(\sum_{j=1}^{n} p_{j} V_{j}^{*} T_{j}\right) x, x\right\rangle\right|^{2}
\end{aligned}
$$

and $\beta(\cdot ; \mathbf{T}, \mathbf{V}, x): \mathbb{R}_{+}^{* n} \rightarrow[0, \infty)$ by

$$
\beta(\cdot ; \mathbf{T}, \mathbf{V}, x)=[\delta(\mathbf{p} ; \mathbf{T}, \mathbf{V}, x)]^{1 / 2} .
$$

Utilising the results from the above section we then have the following vector operator inequalities:

Theorem 6. Let $\mathbf{T}=\left(T_{1}, \ldots, T_{n}\right), \mathbf{V}=\left(V_{1}, \ldots, V_{n}\right) \in \mathcal{B}^{(n)}(H) \backslash\{\mathbf{0}\}$ and $x \in H$.

(i) For any $\mathbf{p}, \mathbf{q} \in \mathbb{R}_{+}^{* n}$ we have

$$
\begin{gathered}
\sigma(\mathbf{p}+\mathbf{q} ; \mathbf{T}, \mathbf{V}, x) \geq \sigma(\mathbf{p} ; \mathbf{T}, \mathbf{V}, x)+\sigma(\mathbf{q} ; \mathbf{T}, \mathbf{V}, x)(\geq 0) \\
\delta(\mathbf{p}+\mathbf{q} ; \mathbf{T}, \mathbf{V}, x)-\delta(\mathbf{p} ; \mathbf{T}, \mathbf{V}, x)-\delta(\mathbf{q} ; \mathbf{T}, \mathbf{V}, x) \\
\quad \geq\left(\operatorname{det}\left[\begin{array}{rr}
\langle\mathbf{T}, \mathbf{T}\rangle_{\mathbf{p}, x}^{1 / 2} & \langle\mathbf{V}, \mathbf{V}\rangle_{\mathbf{p}, x}^{1 / 2} \\
\langle\mathbf{T}, \mathbf{T}\rangle_{\mathbf{q}, x}^{1 / 2} & \langle\mathbf{V}, \mathbf{V}\rangle_{\mathbf{q}, x}^{1 / 2}
\end{array}\right]\right)^{2}(\geq 0)
\end{gathered}
$$

and

$$
\beta(\mathbf{p}+\mathbf{q} ; \mathbf{T}, \mathbf{V}, x) \geq \beta(\mathbf{p} ; \mathbf{T}, \mathbf{V}, x)+\beta(\mathbf{q} ; \mathbf{T}, \mathbf{V}, x)(\geq 0) ;
$$

showing that the functionals $\sigma(\cdot ; \mathbf{T}, \mathbf{V}, x), \delta(\cdot ; \mathbf{T}, \mathbf{V}, x)$ and $\beta(\cdot ; \mathbf{T}, \mathbf{V}, x)$ are superadditive as functions of weights.

(ii) If $\mathbf{p}, \mathbf{q} \in \mathbb{R}_{+}^{* n}$ with $\mathbf{p} \geq \mathbf{q}$, i.e. $p_{i} \geq q_{i}$ for all $i \in\{1, \ldots, n\}$, then

$$
\sigma(\mathbf{p} ; \mathbf{T}, \mathbf{V}, x) \geq \sigma(\mathbf{q} ; \mathbf{T}, \mathbf{V}, x)(\geq 0)
$$

and

$$
\begin{aligned}
& \delta(\mathbf{p} ; \mathbf{T}, \mathbf{V}, x)-\delta(\mathbf{q} ; \mathbf{T}, \mathbf{V}, x) \\
& \geq\left(\operatorname{det}\left[\begin{array}{cc}
\langle\mathbf{T}, \mathbf{T}\rangle_{\mathbf{p}-\mathbf{q}, x}^{1 / 2} & \langle\mathbf{V}, \mathbf{V}\rangle_{\mathbf{p}-\mathbf{q}, x}^{1 / 2} \\
\langle\mathbf{T}, \mathbf{T}\rangle_{\mathbf{q}, x}^{1 / 2} & \langle\mathbf{V}, \mathbf{V}\rangle_{\mathbf{q}, x}^{1 / 2}
\end{array}\right]\right)^{2}(\geq 0)
\end{aligned}
$$

showing that the functionals $\sigma(\cdot ; \mathbf{T}, \mathbf{V}, x)$ and $\delta(\cdot ; \mathbf{T}, \mathbf{V}, x)$ are nondecreasing as functions of weights. 
Similar inequalities may be stated by the use of the rest of the inequalities for Hermitian forms from the above section, namely (2.8)-(2.10). However the details are not presented here.

For other inequalities in inner product spaces and operators on Hilbert spaces see [4]-[25] and the references therein.

\section{Applications for Functions of Normal Operators}

The following result holds:

Theorem 7. Let $f(z):=\sum_{j=0}^{\infty} p_{j} z^{j}$ a power series with nonnegative coefficients and convergent on the open disk $D(0, R), R>0$. If $T$ and $V$ are two normal and commuting operators with $\|T\|^{2},\|V\|^{2}<R$, then we have the inequalities

$$
|\langle f(V T) x, x\rangle| \leq\left\langle f\left(|T|^{2}\right) x, x\right\rangle^{1 / 2}\left\langle f\left(|V|^{2}\right) x, x\right\rangle^{1 / 2}
$$

and

$$
\begin{aligned}
& \left|\left\langle f\left(|T|^{2}\right) x, x\right\rangle+\langle f(V T) x, x\rangle+\left\langle f\left(V^{*} T^{*}\right) x, x\right\rangle+\left\langle f\left(|V|^{2}\right) x, x\right\rangle\right|^{1 / 2} \\
& \leq\left\langle f\left(|T|^{2}\right) x, x\right\rangle^{1 / 2}+\left\langle f\left(|V|^{2}\right) x, x\right\rangle^{1 / 2}
\end{aligned}
$$

for any $x \in H$.

Proof. If we use the inequality (3.4) for powers of operators we have

$$
\left|\left\langle\sum_{j=0}^{m} p_{j} V^{j} T^{j} x, x\right\rangle\right|^{2} \leq\left\langle\sum_{j=0}^{m} p_{j}\left|T^{j}\right|^{2} x, x\right\rangle\left\langle\sum_{j=0}^{m} p_{j}\left|\left(V^{*}\right)^{j}\right|^{2} x, x\right\rangle
$$

for any $m \geq 1$ and $x \in H$.

Since $V$ and $T$ are normal we have $\left|T^{j}\right|^{2}=|T|^{2 j}$ and $\left|\left(V^{*}\right)^{j}\right|^{2}=|V|^{2 j}$ for any $j \in\{0, \ldots, m\}$ and by the commutativity of $V$ and $T$ we also have $V^{j} T^{j}=(V T)^{j}$ for any $j \in\{0, \ldots, m\}$.

Therefore we can state that

$$
\left|\left\langle\sum_{j=0}^{m} p_{j}(V T)^{j} x, x\right\rangle\right|^{2} \leq\left\langle\sum_{j=0}^{m} p_{j}|T|^{2 j} x, x\right\rangle\left\langle\sum_{j=0}^{m} p_{j}|V|^{2 j} x, x\right\rangle
$$

for any $m \geq 1$ and $x \in H$. 
Since all the series whose partial sums are involved in the inequality (4.4) are convergent then by letting $m \rightarrow \infty$ in (4.4) we get (4.1).

By the normality and commutativity of $V$ and $T$ we have

$$
\sum_{j=0}^{m} p_{j}\left|T^{j}+\left(V^{*}\right)^{j}\right|^{2}=\sum_{j=0}^{m} p_{j}\left[|T|^{2 j}+(V T)^{j}+\left(V^{*} T^{*}\right)^{j}+|V|^{2 j}\right] .
$$

Then from (4.2) we have

$$
\begin{aligned}
& \left\langle\sum_{j=0}^{m} p_{j}\left[|T|^{2 j}+(V T)^{j}+\left(V^{*} T^{*}\right)^{j}+|V|^{2 j}\right] x, x\right\rangle^{1 / 2} \\
& \leq\left\langle\left(\sum_{j=0}^{m} p_{j}|T|^{2 j}\right) x, x\right\rangle^{1 / 2}+\left\langle\left(\sum_{j=0}^{m} p_{j}|V|^{2 j}\right) x, x\right\rangle^{1 / 2}
\end{aligned}
$$

for any $m \geq 1$ and $x \in H$.

Since all the series whose partial sums are involved in the inequality (4.5) are convergent then by letting $m \rightarrow \infty$ in (4.5) we get (4.2).

By utilizing Theorem 6 and a similar argument to the one from Theorem 7 we also have:

Theorem 8. Let $f(z):=\sum_{j=0}^{\infty} p_{j} z^{j}$ and $g(z):=\sum_{j=0}^{\infty} q_{j} z^{j}$ be two power series with nonnegative coefficients and convergent on the open disk $D(0, R)$, $R>0$. If $T$ and $V$ are two normal and commuting operators with $\|T\|^{2},\|V\|^{2}<$ $R$ and if we define the functionals

$$
\begin{gathered}
\sigma(f ; T, V, x):=\left\langle f\left(|T|^{2}\right) x, x\right\rangle^{1 / 2}\left\langle f\left(|V|^{2}\right) x, x\right\rangle^{1 / 2}-|\langle f(V T) x, x\rangle|, \\
\delta(f ; T, V, x):=\left\langle f\left(|T|^{2}\right) x, x\right\rangle\left\langle f\left(|V|^{2}\right) x, x\right\rangle-|\langle f(V T) x, x\rangle|^{2}
\end{gathered}
$$

and

$$
\beta(f ; T, V, x):=\left[\left\langle f\left(|T|^{2}\right) x, x\right\rangle\left\langle f\left(|V|^{2}\right) x, x\right\rangle-|\langle f(V T) x, x\rangle|^{2}\right]^{1 / 2}
$$

where $x \in H$, then

$$
\begin{aligned}
& \sigma(f+g ; T, V, x) \geq \sigma(f ; T, V, x)+\sigma(g ; T, V, x) \quad(\geq 0), \\
\delta & (f+g ; T, V, x)-\delta(f ; T, V, x)-\delta(g ; T, V, x) \\
\geq & \left(\operatorname{det}\left[\begin{array}{cc}
\left\langle f\left(|T|^{2}\right) x, x\right\rangle^{1 / 2} & \left\langle f\left(|V|^{2}\right) x, x\right\rangle^{1 / 2} \\
\left\langle g\left(|T|^{2}\right) x, x\right\rangle^{1 / 2} & \left\langle g\left(|V|^{2}\right) x, x\right\rangle^{1 / 2}
\end{array}\right]\right)^{2}(\geq 0)
\end{aligned}
$$


and

$$
\beta(f+g ; T, V, x) \geq \beta(f ; T, V, x)+\beta(g ; T, V, x)(\geq 0)
$$

for any $x \in H$.

Moreover, if $p_{j} \geq q_{j}$ for all $j \in \mathbb{N}$, then we have

$$
\sigma(f ; T, V, x) \geq \sigma(g ; T, V, x) \quad(\geq 0),
$$

and

$$
\begin{aligned}
& \delta(f ; T, V, x)-\delta(g ; T, V, x) \\
& \geq\left(\operatorname{det}\left[\begin{array}{ll}
\left\langle f\left(|T|^{2}\right) x, x\right\rangle^{1 / 2} & \left\langle f\left(|V|^{2}\right) x, x\right\rangle^{1 / 2} \\
\left\langle(f-g)\left(|T|^{2}\right) x, x\right\rangle^{1 / 2} & \left\langle(f-g)\left(|V|^{2}\right) x, x\right\rangle^{1 / 2}
\end{array}\right]\right)^{2}(\geq 0)
\end{aligned}
$$

for any $x \in H$.

Some important examples of power series with nonnegative coefficients are

$$
\begin{aligned}
\frac{1}{1-\lambda} & =\sum_{n=0}^{\infty} \lambda^{n}, \lambda \in D(0,1) ; \\
\ln \frac{1}{1-\lambda} & =\sum_{n=1}^{\infty} \frac{1}{n} \lambda^{n}, \lambda \in D(0,1) ; \\
\exp (\lambda) & =\sum_{n=0}^{\infty} \frac{1}{n !} \lambda^{n}, \lambda \in \mathbb{C} ; \\
\sinh \lambda & =\sum_{n=0}^{\infty} \frac{1}{(2 n+1) !} \lambda^{2 n+1}, \lambda \in \mathbb{C} ; \\
\cosh \lambda & =\sum_{n=0}^{\infty} \frac{1}{(2 n) !} \lambda^{2 n}, \lambda \in \mathbb{C} .
\end{aligned}
$$

Other important examples of functions as power series representations with 
nonnegative coefficients are:

$$
\begin{aligned}
\frac{1}{2} \ln \left(\frac{1+\lambda}{1-\lambda}\right) & =\sum_{n=1}^{\infty} \frac{1}{2 n-1} \lambda^{2 n-1}, \lambda \in D(0,1) \\
\sin ^{-1}(\lambda) & =\sum_{n=0}^{\infty} \frac{\Gamma\left(n+\frac{1}{2}\right)}{\sqrt{\pi}(2 n+1) n !} \lambda^{2 n+1}, \lambda \in D(0,1) \\
\tanh ^{-1}(\lambda) & =\sum_{n=1}^{\infty} \frac{1}{2 n-1} \lambda^{2 n-1}, \lambda \in D(0,1) \\
{ }_{2} F_{1}(\alpha, \beta, \gamma, \lambda) & =\sum_{n=0}^{\infty} \frac{\Gamma(n+\alpha) \Gamma(n+\beta) \Gamma(\gamma)}{n ! \Gamma(\alpha) \Gamma(\beta) \Gamma(n+\gamma)} \lambda^{n}, \alpha, \beta, \gamma>0 \\
\lambda & \in D(0,1)
\end{aligned}
$$

where $\Gamma$ is Gamma function.

If $T$ and $V$ are two normal and commuting operators with $\|T\|,\|V\|<1$, then we have the inequalities

$$
\begin{aligned}
& \left|\left\langle\left(1_{H}-V T\right)^{-1} x, x\right\rangle\right| \\
& \leq\left\langle\left(1_{H}-|T|^{2}\right)^{-1} x, x\right\rangle^{1 / 2}\left\langle\left(1_{H}-|V|^{2}\right)^{-1} x, x\right\rangle^{1 / 2}
\end{aligned}
$$

and

$$
\begin{aligned}
& \left|\left\langle\ln \left(1_{H}-V T\right)^{-1} x, x\right\rangle\right| \\
& \leq\left\langle\ln \left(1_{H}-|T|^{2}\right)^{-1} x, x\right\rangle^{1 / 2}\left\langle\ln \left(1_{H}-|V|^{2}\right)^{-1} x, x\right\rangle^{1 / 2}
\end{aligned}
$$

for any $x \in H, x \neq 0$.

For any $T$ and $V$ normal and commuting operators we have

$$
\begin{gathered}
|\langle\exp (V T) x, x\rangle| \leq\left\langle\exp \left(|T|^{2}\right) x, x\right\rangle^{1 / 2}\left\langle\exp \left(|V|^{2}\right) x, x\right\rangle^{1 / 2}, \\
|\langle\sinh (V T) x, x\rangle| \leq\left\langle\sinh \left(|T|^{2}\right) x, x\right\rangle^{1 / 2}\left\langle\sinh \left(|V|^{2}\right) x, x\right\rangle^{1 / 2}
\end{gathered}
$$

and

$$
|\langle\cosh (V T) x, x\rangle| \leq\left\langle\cosh \left(|T|^{2}\right) x, x\right\rangle^{1 / 2}\left\langle\cosh \left(|V|^{2}\right) x, x\right\rangle^{1 / 2}
$$

for any $x \in H$.

Now, observe that if we take

$$
f(\lambda)=\sinh \lambda=\sum_{n=0}^{\infty} \frac{1}{(2 n+1) !} \lambda^{2 n+1}
$$


and

$$
g(\lambda)=\cosh \lambda=\sum_{n=0}^{\infty} \frac{1}{(2 n) !} \lambda^{2 n}
$$

then $f(\lambda)+g(\lambda)=\exp \lambda$ for any $\lambda \in \mathbb{C}$.

If we use the superadditivity properties from (4.6)-(4.8) we have for any $T$ and $V$ normal and commuting operators

$$
\begin{aligned}
\left\langle\exp \left(|T|^{2}\right) x, x\right\rangle^{1 / 2}\left\langle\exp \left(|V|^{2}\right) x, x\right\rangle^{1 / 2}-|\langle\exp (V T) x, x\rangle| \\
\geq\left\langle\sinh \left(|T|^{2}\right) x, x\right\rangle^{1 / 2}\left\langle\sinh \left(|V|^{2}\right) x, x\right\rangle^{1 / 2}-|\langle\sinh (V T) x, x\rangle| \\
+\left\langle\cosh \left(|T|^{2}\right) x, x\right\rangle^{1 / 2}\left\langle\cosh \left(|V|^{2}\right) x, x\right\rangle^{1 / 2}-|\langle\cosh (V T) x, x\rangle|(\geq 0), \\
\quad\left\langle\exp \left(|T|^{2}\right) x, x\right\rangle\left\langle\exp \left(|V|^{2}\right) x, x\right\rangle-|\langle\exp (V T) x, x\rangle|^{2} \\
\quad-\left\langle\sinh \left(|T|^{2}\right) x, x\right\rangle\left\langle\sinh \left(|V|^{2}\right) x, x\right\rangle+|\langle\sinh (V T) x, x\rangle|^{2} \\
\quad-\left\langle\cosh \left(|T|^{2}\right) x, x\right\rangle\left\langle\cosh \left(|V|^{2}\right) x, x\right\rangle+|\langle f(V T) x, x\rangle|^{2} \\
\geq\left(\operatorname{det}\left[\begin{array}{ll}
\left\langle\sinh \left(|T|^{2}\right) x, x\right\rangle^{1 / 2} & \left\langle\sinh \left(|V|^{2}\right) x, x\right\rangle^{1 / 2} \\
\left\langle\cosh \left(|T|^{2}\right) x, x\right\rangle^{1 / 2} & \left\langle\cosh \left(|V|^{2}\right) x, x\right\rangle^{1 / 2}
\end{array}\right]\right)^{2}(\geq 0)
\end{aligned}
$$

and

$$
\begin{aligned}
& {\left[\left\langle\exp \left(|T|^{2}\right) x, x\right\rangle\left\langle\exp \left(|V|^{2}\right) x, x\right\rangle-|\langle\exp (V T) x, x\rangle|^{2}\right]^{1 / 2}} \\
& \geq\left[\left\langle\sinh \left(|T|^{2}\right) x, x\right\rangle\left\langle\sinh \left(|V|^{2}\right) x, x\right\rangle-|\langle\sinh (V T) x, x\rangle|^{2}\right]^{1 / 2} \\
& +\left[\left\langle\cosh \left(|T|^{2}\right) x, x\right\rangle\left\langle\cosh \left(|V|^{2}\right) x, x\right\rangle-|\langle\cosh (V T) x, x\rangle|^{2}\right]^{1 / 2}(\geq 0)
\end{aligned}
$$

for any $x \in H$.

Now, consider the series $\frac{1}{1-\lambda}=\sum_{n=0}^{\infty} \lambda^{n}, \lambda \in D(0,1)$ and $\ln \frac{1}{1-\lambda}=$ $\sum_{n=1}^{\infty} \frac{1}{n} \lambda^{n}, \lambda \in D(0,1)$ and define $p_{n}=1, n \geq 0, q_{0}=0, q_{n}=\frac{1}{n}, n \geq 1$ then we observe that for any $n \geq 0$ we have $p_{n} \geq q_{n}$.

Making use of the monotonicity properties from (4.9) and (4.10) we can state that

$$
\begin{aligned}
& \left\langle\left(1_{H}-|T|^{2}\right)^{-1} x, x\right\rangle^{1 / 2}\left\langle\left(1_{H}-|V|^{2}\right)^{-1} x, x\right\rangle^{1 / 2} \\
& -\left|\left\langle\left(1_{H}-V T\right)^{-1} x, x\right\rangle\right| \\
& \geq\left\langle\ln \left(1_{H}-|T|^{2}\right)^{-1} x, x\right\rangle^{1 / 2}\left\langle\ln \left(1_{H}-|V|^{2}\right)^{-1} x, x\right\rangle^{1 / 2} \\
& -\left|\left\langle\ln \left(1_{H}-V T\right)^{-1} x, x\right\rangle\right|(\geq 0)
\end{aligned}
$$


and

$$
\begin{aligned}
& \left\langle\left(1_{H}-|T|^{2}\right)^{-1} x, x\right\rangle\left\langle\left(1_{H}-|V|^{2}\right)^{-1} x, x\right\rangle \\
& -\left|\left\langle\left(1_{H}-V T\right)^{-1} x, x\right\rangle\right|^{2} \\
& \geq\left\langle\ln \left(1_{H}-|T|^{2}\right)^{-1} x, x\right\rangle\left\langle\ln \left(1_{H}-|V|^{2}\right)^{-1} x, x\right\rangle \\
& -\left|\left\langle\ln \left(1_{H}-V T\right)^{-1} x, x\right\rangle\right|^{2}(\geq 0)
\end{aligned}
$$

for any $T$ and $V$ two normal and commuting operators with $\|T\|,\|V\|<1$ and $x \in H$.

\section{Applications for One and Two Operators}

If we write the inequality (3.4) for $p_{j}=1, j \in\{1, \ldots, n\}$ then we have

$$
\left|\left\langle\left(\sum_{j=1}^{n} V_{j}^{*} T_{j}\right) x, x\right\rangle\right|^{2} \leq\left\langle\left(\sum_{j=1}^{n}\left|T_{j}\right|^{2}\right) x, x\right\rangle\left\langle\left(\sum_{j=1}^{n}\left|V_{j}\right|^{2}\right) x, x\right\rangle
$$

for any $x \in H$.

If we take in this $n=2$ we get

$$
\left|\left\langle\left(V_{1}^{*} T_{1}+V_{2}^{*} T_{2}\right) x, x\right\rangle\right|^{2} \leq\left\langle\left(\left|T_{1}\right|^{2}+\left|T_{2}\right|^{2}\right) x, x\right\rangle\left\langle\left(\left|V_{1}\right|^{2}+\left|V_{2}\right|^{2}\right) x, x\right\rangle
$$

for any $T_{1}, T_{2}, V_{1}, V_{2} \in \mathcal{B}(H)$ and any $x \in H$.

If we take $T=(A, B)$ and $V=\left(B^{*}, \pm A^{*}\right)$ in (5.2), where $A, B \in \mathcal{B}(H)$, then we have

$$
|\langle(B A \pm A B) x, x\rangle|^{2} \leq\left\langle\left(|A|^{2}+|B|^{2}\right) x, x\right\rangle\left\langle\left(\left|A^{*}\right|^{2}+\left|B^{*}\right|^{2}\right) x, x\right\rangle
$$

for any $x \in H$.

In particular, for $B=A^{*}$ in (5.3) we get

$$
\left|\left\langle\left(A^{*} A-A A^{*}\right) x, x\right\rangle\right| \leq\left\langle\left(|A|^{2}+\left|A^{*}\right|^{2}\right) x, x\right\rangle
$$

for any $x \in H$.

If we take in (5.2) $T=(C, I)$ and $V=\left(I, \pm D^{*}\right)$ where $C, D \in \mathcal{B}(H)$, then we get

$$
|\langle(C \pm D) x, x\rangle|^{2} \leq\left\langle\left(|C|^{2}+I\right) x, x\right\rangle\left\langle\left(\left|D^{*}\right|^{2}+I\right) x, x\right\rangle
$$


for any $x \in H$, and by taking in this inequality $C=B A$ and $D=A B$ where $A, B \in \mathcal{B}(H)$, then we get

$$
|\langle(B A \pm A B) x, x\rangle|^{2} \leq\left\langle\left(|B A|^{2}+I\right) x, x\right\rangle\left\langle\left(\left|B^{*} A^{*}\right|^{2}+I\right) x, x\right\rangle
$$

for any $x \in H$. In particular, if $B=A^{*}$, then

$$
\left|\left\langle\left(A^{*} A \pm A A^{*}\right) x, x\right\rangle\right|^{2} \leq\left\langle\left(|A|^{4}+I\right) x, x\right\rangle\left\langle\left(\left|A^{*}\right|^{4}+I\right) x, x\right\rangle
$$

for any $x \in H$.

Moreover, if we choose $T=(B, I)$ and $V=\left(A^{*},(A B)^{*}\right)$ in (5.2), where $A, B \in \mathcal{B}(H)$, then we get

$$
|\langle A B x, x\rangle|^{2} \leq\left\langle\left(\frac{|B|^{2}+I}{2}\right) x, x\right\rangle\left\langle\left(\frac{\left|A^{*}\right|^{2}+\left|(A B)^{*}\right|^{2}}{2}\right) x, x\right\rangle
$$

for any $x \in H$.

Since

$$
\left|A^{*}\right|^{2}+\left|(A B)^{*}\right|^{2}=A A^{*}+A B B^{*} A^{*}=A\left(I+\left|B^{*}\right|^{2}\right) A^{*}
$$

then we have

$$
|\langle A B x, x\rangle|^{2} \leq\left\langle\left(\frac{|B|^{2}+I}{2}\right) x, x\right\rangle\left\langle\left(A\left(\frac{I+\left|B^{*}\right|^{2}}{2}\right) A^{*}\right) x, x\right\rangle
$$

for any $x \in H$.

Moreover, if we take in (5.8) $A=I$, then we get

$$
|\langle B x, x\rangle|^{2} \leq\left\langle\left(\frac{|B|^{2}+I}{2}\right) x, x\right\rangle\left\langle\left(\frac{I+\left|B^{*}\right|^{2}}{2}\right) x, x\right\rangle
$$

for any $B \in \mathcal{B}(H)$ and for any $x \in H$.

Also, if we choose $A=B$ in (5.8), then we get

$$
\left|\left\langle B^{2} x, x\right\rangle\right| \leq\left\langle\left(\frac{|B|^{2}+I}{2}\right) x, x\right\rangle^{1 / 2}\left\langle B\left(\frac{I+\left|B^{*}\right|^{2}}{2}\right) B^{*} x, x\right\rangle^{1 / 2}
$$

while for $A=B^{*}$ we get

$$
\|B x\|^{2} \leq\left\langle\left(\frac{|B|^{2}+I}{2}\right) x, x\right\rangle^{1 / 2}\left\langle B^{*}\left(\frac{I+\left|B^{*}\right|^{2}}{2}\right) B x, x\right\rangle^{1 / 2}
$$


for any $B \in \mathcal{B}(H)$ and for any $x \in H$.

Let $C=A+i B$ be the Cartesian decomposition of the operator $C$. Then $A$ and $B$ are selfadjoint and

$$
A^{2}+B^{2}=\frac{1}{2}\left(C^{*} C+C C^{*}\right)=\frac{1}{2}\left(|C|^{2}+\left|C^{*}\right|^{2}\right) .
$$

Moreover

$$
A=\frac{C+C^{*}}{2}=: \operatorname{Re} C \text { and } B=\frac{C-C^{*}}{2 i}=: \operatorname{Im} C .
$$

If we apply the inequality (5.2) for $T=(A, i I), V=(I, B)$ then we get

$$
|\langle C x, x\rangle| \leq\left\langle\left(|\operatorname{Re} C|^{2}+I\right) x, x\right\rangle^{1 / 2}\left\langle\left(|\operatorname{Im} C|^{2}+I\right) x, x\right\rangle^{1 / 2},
$$

for any $C \in \mathcal{B}(H)$ and for any $x \in H$.

\section{Other Inequalities for Sums}

On utilizing the inequality for two operators above we can obtain other inequalities for sums as follows:

Theorem 9. For any $\left(p_{1}, \ldots, p_{n}\right) \in \mathbb{R}_{+}^{* n}, \quad\left(A_{1}, \ldots, A_{n}\right),\left(B_{1}, \ldots, B_{n}\right) \in \mathcal{B}^{(n)}(H)$ and $x \in H$ we have the inequality

$$
\begin{aligned}
& \left|\left\langle\left(\sum_{j=1}^{n} p_{j} A_{j} B_{j}\right) x, x\right\rangle\right|^{2} \\
& \leq\left\langle\sum_{j=1}^{n} p_{j}\left(\frac{|B|_{j}^{2}+I}{2}\right) x, x\right\rangle\left\langle\sum_{j=1}^{n} p_{j}\left(A_{j}\left(\frac{I+\left|B_{j}^{*}\right|^{2}}{2}\right) A_{j}^{*}\right) x, x\right\rangle .
\end{aligned}
$$

Proof. Applying the inequality (5.8) we have

$$
\left|\left\langle A_{j} B_{j} x, x\right\rangle\right| \leq\left\langle\left(\frac{\left|B_{j}\right|^{2}+I}{2}\right) x, x\right\rangle^{1 / 2}\left\langle\left(A_{j}\left(\frac{I+\left|B_{j}^{*}\right|^{2}}{2}\right) A_{j}^{*}\right) x, x\right\rangle^{1 / 2}
$$

for any $x \in H$ and $j \in\{1, \ldots, n\}$. 
Using the generalized triangle inequality for modulus, the inequality (6.2) and the weighted Cauchy-Bunyakowsky-Schwarz inequality we have

$$
\begin{aligned}
& \left|\left\langle\left(\sum_{j=1}^{n} p_{j} A_{j} B_{j}\right) x, x\right\rangle\right| \\
& =\left|\sum_{j=1}^{n} p_{j}\left\langle A_{j} B_{j} x, x\right\rangle\right| \leq \sum_{j=1}^{n} p_{j}\left|\left\langle A_{j} B_{j} x, x\right\rangle\right| \\
& \leq \sum_{j=1}^{n} p_{j}\left\langle\left(\frac{\left|B_{j}\right|^{2}+I}{2}\right) x, x\right\rangle^{1 / 2}\left\langle\left(A_{j}\left(\frac{I+\left|B_{j}^{*}\right|^{2}}{2}\right) A_{j}^{*}\right) x, x\right\rangle^{1 / 2} \\
& \leq\left[\sum_{j=1}^{n} p_{j}\left(\left\langle\left(\frac{\left|B_{j}\right|^{2}+I}{2}\right) x, x\right\rangle^{1 / 2}\right)^{2}\right]^{1 / 2} \\
& \times\left[\sum_{j=1}^{n} p_{j}\left(\left\langle\left(A_{j}\left(\frac{I+\left|B_{j}^{*}\right|^{2}}{2}\right) A_{j}^{*}\right) x, x\right\rangle^{1 / 2}\right)^{2}\right]^{1 / 2} \\
& =\left\langle\sum_{j=1}^{n} p_{j}\left(\frac{|B|_{j}^{2}+I}{2}\right) x, x\right\rangle\left\langle\sum_{j=1}^{n} p_{j}\left(A_{j}\left(\frac{I+\left|B_{j}^{*}\right|^{2}}{2}\right) A_{j}^{*}\right) x, x\right\rangle
\end{aligned}
$$

for any $x \in H$ and the inequality (6.1) is proved.

Corollary 3. For any $\left(p_{1}, \ldots, p_{n}\right) \in \mathbb{R}_{+}^{* n}, \quad\left(B_{1}, \ldots, B_{n}\right) \in \mathcal{B}^{(n)}(H)$ and $x \in H$ we have the inequality

$$
\begin{aligned}
& \left|\left\langle\left(\sum_{j=1}^{n} p_{j} B_{j}\right) x, x\right\rangle\right|^{2} \\
& \leq\left\langle\sum_{j=1}^{n} p_{j}\left(\frac{|B|_{j}^{2}+I}{2}\right) x, x\right\rangle\left\langle\sum_{j=1}^{n} p_{j}\left(\left(\frac{I+\left|B_{j}^{*}\right|^{2}}{2}\right)\right) x, x\right\rangle \\
& \left|\left\langle\left(\sum_{j=1}^{n} p_{j} B_{j}^{2}\right) x, x\right\rangle\right|^{2} \\
& \leq\left\langle\sum_{j=1}^{n} p_{j}\left(\frac{|B|_{j}^{2}+I}{2}\right) x, x\right\rangle\left\langle\sum_{j=1}^{n} p_{j}\left(B_{j}\left(\frac{I+\left|B_{j}^{*}\right|^{2}}{2}\right) B_{j}^{*}\right) x, x\right\rangle,
\end{aligned}
$$




$$
\begin{aligned}
& \left|\left\langle\left(\sum_{j=1}^{n} p_{j}|B|_{j}^{2}\right) x, x\right\rangle\right|^{2} \\
& \leq\left\langle\sum_{j=1}^{n} p_{j}\left(\frac{|B|_{j}^{2}+I}{2}\right) x, x\right\rangle\left\langle\sum_{j=1}^{n} p_{j}\left(B_{j}^{*}\left(\frac{I+\left|B_{j}^{*}\right|^{2}}{2}\right) B_{j}\right) x, x\right\rangle
\end{aligned}
$$

and

$$
\begin{aligned}
& \left|\left\langle\left(\sum_{j=1}^{n} p_{j} B_{j}\right) x, x\right\rangle\right|^{2} \\
& \leq\left\langle\sum_{j=1}^{n} p_{j}\left(\left|\operatorname{Re} B_{j}\right|^{2}+I\right) x, x\right\rangle\left\langle\sum_{j=1}^{n} p_{j}\left(\left|\operatorname{Im} B_{j}\right|^{2}+I\right) x, x\right\rangle .
\end{aligned}
$$

\section{Inequalities for Numerical Radius}

The numerical radius $w(T)$ of an operator $T$ on $H$ is given by $[26$, p. 8]:

$$
w(T)=\sup \{|\lambda|, \lambda \in W(T)\}=\sup \{|\langle T x, x\rangle|,\|x\|=1\} .
$$

It is well known that $w(\cdot)$ is a norm on the Banach algebra $\mathcal{B}(H)$ of all bounded linear operators $T: H \rightarrow H$. This norm is equivalent with the operator norm. In fact, the following more precise result holds [26, p. 9]:

Theorem 10 (Equivalent norm). For any $T \in \mathcal{B}(H)$ one has

$$
w(T) \leq\|T\| \leq 2 w(T) .
$$

We recall also that if $T$ is normal operator, then $w(T)=\|T\|$.

For a survey of recent inequalities for numerical radius, see [20] and the references therein.

We have the following result:

Theorem 11. For any $\left(p_{1}, \ldots, p_{n}\right) \in \mathbb{R}_{+}^{* n},\left(V_{1}, \ldots, V_{n}\right),\left(T_{1}, \ldots, T_{n}\right) \in \mathcal{B}^{(n)}(H)$ we have the inequalities

$$
w^{2}\left(\sum_{j=1}^{n} p_{j} V_{j}^{*} T_{j}\right) \leq\left\|\sum_{j=1}^{n} p_{j}\left|T_{j}\right|^{2}\right\|\left\|\sum_{j=1}^{n} p_{j}\left|V_{j}\right|^{2}\right\|
$$

and

$$
w^{2}\left(\sum_{j=1}^{n} p_{j} V_{j}^{*} T_{j}\right) \leq\left\|\alpha\left(\sum_{j=1}^{n} p_{j}\left|T_{j}\right|^{2}\right)^{\frac{1}{\alpha}}+(1-\alpha)\left(\sum_{j=1}^{n} p_{j}\left|V_{j}\right|^{2}\right)^{\frac{1}{1-\alpha}}\right\|
$$


for $\alpha \in(0,1)$.

Proof. Taking the supremum over $\|x\|=1$ in (3.4) we have

$$
\begin{aligned}
& \sup _{\|x\|=1}\left|\left\langle\left(\sum_{j=1}^{n} p_{j} V_{j}^{*} T_{j}\right) x, x\right\rangle\right|^{2} \\
& \leq \sup _{\|x\|=1}\left\{\left\langle\left(\sum_{j=1}^{n} p_{j}\left|T_{j}\right|^{2}\right) x, x\right\rangle\left\langle\left(\sum_{j=1}^{n} p_{j}\left|V_{j}\right|^{2}\right) x, x\right\rangle\right\} \\
& \leq \sup _{\|x\|=1}\left\langle\left(\sum_{j=1}^{n} p_{j}\left|T_{j}\right|^{2}\right) x, x\right\rangle \sup _{\|x\|=1}\left\langle\left(\sum_{j=1}^{n} p_{j}\left|V_{j}\right|^{2}\right) x, x\right\rangle .
\end{aligned}
$$

Since

$$
\begin{gathered}
\sup _{\|x\|=1}\left|\left\langle\left(\sum_{j=1}^{n} p_{j} V_{j}^{*} T_{j}\right) x, x\right\rangle\right|^{2}=w^{2}\left(\sum_{j=1}^{n} p_{j} A_{j} B_{j}\right), \\
\sup _{\|x\|=1}\left\langle\left(\sum_{j=1}^{n} p_{j}\left|T_{j}\right|^{2}\right) x, x\right\rangle=\left\|\sum_{j=1}^{n} p_{j}\left|T_{j}\right|^{2}\right\|
\end{gathered}
$$

and

$$
\sup _{\|x\|=1}\left\langle\left(\sum_{j=1}^{n} p_{j}\left|V_{j}\right|^{2}\right) x, x\right\rangle=\left\|\sum_{j=1}^{n} p_{j}\left|V_{j}\right|^{2}\right\|,
$$

then by (7.5) we deduce the desired result (7.3).

If $p \in(0,1)$, then we have the following inequality for positive operators $P \geq 0$

$$
\left\langle P^{p} x, x\right\rangle \leq\langle P x, x\rangle^{p} \text { for any } x \in H,\|x\|=1 \text {. }
$$

Therefore, if $\alpha \in(0,1)$ then

$$
\begin{aligned}
\left\langle\left(\sum_{j=1}^{n} p_{j}\left|T_{j}\right|^{2}\right) x, x\right\rangle & =\left\langle\left[\left(\sum_{j=1}^{n} p_{j}\left|T_{j}\right|^{2}\right)^{\frac{1}{\alpha}}\right]^{\alpha} x, x\right\rangle \\
& \leq\left\langle\left(\sum_{j=1}^{n} p_{j}\left|T_{j}\right|^{2}\right)^{\frac{1}{\alpha}} x, x\right\rangle^{\alpha}
\end{aligned}
$$


and

$$
\begin{aligned}
\left\langle\left(\sum_{j=1}^{n} p_{j}\left|V_{j}\right|^{2}\right) x, x\right\rangle & =\left\langle\left[\left(\sum_{j=1}^{n} p_{j}\left|V_{j}\right|^{2}\right)^{\frac{1}{1-\alpha}}\right]^{1-\alpha} x, x\right\rangle \\
& \leq\left\langle\left(\sum_{j=1}^{n} p_{j}\left|V_{j}\right|^{2}\right)^{\frac{1}{1-\alpha}} x, x\right\rangle^{1-\alpha}
\end{aligned}
$$

for any $x \in H,\|x\|=1$.

If we multiply these two inequalities and use the weighted arithmetic mean-geometric mean inequality, we have

$$
\begin{aligned}
& \left\langle\left(\sum_{j=1}^{n} p_{j}\left|T_{j}\right|^{2}\right) x, x\right\rangle\left\langle\left(\sum_{j=1}^{n} p_{j}\left|V_{j}\right|^{2}\right) x, x\right\rangle \\
& \leq\left\langle\left(\sum_{j=1}^{n} p_{j}\left|T_{j}\right|^{2}\right)^{\frac{1}{\alpha}} x, x\right\rangle^{\alpha}\left\langle\left(\sum_{j=1}^{n} p_{j}\left|V_{j}\right|^{2}\right)^{\frac{1}{1-\alpha}} x, x\right\rangle^{1-\alpha} \\
& \leq \alpha\left\langle\left(\sum_{j=1}^{n} p_{j}\left|T_{j}\right|^{2}\right)^{\frac{1}{\alpha}} x, x\right\rangle+(1-\alpha)\left\langle\left(\sum_{j=1}^{n} p_{j}\left|V_{j}\right|^{2}\right)^{\frac{1}{1-\alpha}} x, x\right\rangle \\
& =\left\langle\left[\alpha\left(\sum_{j=1}^{n} p_{j}\left|T_{j}\right|^{2}\right)^{\frac{1}{\alpha}}+(1-\alpha)\left(\sum_{j=1}^{n} p_{j}\left|V_{j}\right|^{2}\right)^{\frac{1}{1-\alpha}}\right] x\right\rangle
\end{aligned}
$$

for any $x \in H,\|x\|=1$.

From (3.4) and (7.6) we have

$$
\begin{aligned}
& \left|\left\langle\left(\sum_{j=1}^{n} p_{j} V_{j}^{*} T_{j}\right) x, x\right\rangle\right|^{2} \\
& \leq\left\langle\left[\alpha\left(\sum_{j=1}^{n} p_{j}\left|T_{j}\right|^{2}\right)^{\frac{1}{\alpha}}+(1-\alpha)\left(\sum_{j=1}^{n} p_{j}\left|V_{j}\right|^{2}\right)^{\frac{1}{1-\alpha}}\right] x, x\right\rangle
\end{aligned}
$$

for any $x \in H,\|x\|=1$.

Taking the supremum over $\|x\|=1$ in (7.7) we get (7.4).

In a similar way, by utilizing (6.1) we can prove the following result as well: 
Theorem 12. For any $\left(p_{1}, \ldots, p_{n}\right) \in \mathbb{R}_{+}^{* n}, \quad\left(A_{1}, \ldots, A_{n}\right),\left(B_{1}, \ldots, B_{n}\right) \in \mathcal{B}^{(n)}(H)$ we have the inequality

$$
\begin{aligned}
& w^{2}\left(\sum_{j=1}^{n} p_{j} A_{j} B_{j}\right) \\
& \leq\left\|\sum_{j=1}^{n} p_{j}\left(\frac{|B|_{j}^{2}+I}{2}\right)\right\|\left\|\sum_{j=1}^{n} p_{j}\left(A_{j}\left(\frac{I+\left|B_{j}^{*}\right|^{2}}{2}\right) A_{j}^{*}\right)\right\| .
\end{aligned}
$$

In particular, we have

$$
\begin{aligned}
& w^{2}\left(\sum_{j=1}^{n} p_{j} B_{j}\right) \leq\left\|\sum_{j=1}^{n} p_{j}\left(\frac{|B|_{j}^{2}+I}{2}\right)\right\|\left\|\sum_{j=1}^{n} p_{j}\left(\frac{I+\left|B_{j}^{*}\right|^{2}}{2}\right)\right\|, \\
& w^{2}\left(\sum_{j=1}^{n} p_{j} B_{j}^{2}\right) \\
& \leq\left\|\sum_{j=1}^{n} p_{j}\left(\frac{|B|_{j}^{2}+I}{2}\right)\right\|\left\|\sum_{j=1}^{n} p_{j}\left(B_{j}\left(\frac{I+\left|B_{j}^{*}\right|^{2}}{2}\right) B_{j}^{*}\right)\right\|, \\
& \left\|\sum_{j=1}^{n} p_{j}|B|_{j}^{2}\right\|^{2} \\
& \leq\left\|\sum_{j=1}^{n} p_{j}\left(\frac{|B|_{j}^{2}+I}{2}\right)\right\|\left\|\sum_{j=1}^{n} p_{j}\left(B_{j}^{*}\left(\frac{I+\left|B_{j}^{*}\right|^{2}}{2}\right) B_{j}\right)\right\|
\end{aligned}
$$

and

$$
w^{2}\left(\sum_{j=1}^{n} p_{j} B_{j}\right) \leq\left\|\sum_{j=1}^{n} p_{j}\left(\left|\operatorname{Re} B_{j}\right|^{2}+I\right)\right\|\left\|\sum_{j=1}^{n} p_{j}\left(\left|\operatorname{Im} B_{j}\right|^{2}+I\right)\right\| .
$$

Utilising the above results we can state various inequalities for power series of normal operators. However the details are not presented here.

\section{References}

[1] N. G. de Bruijn, Problem 12, Wisk. Opgaven, 21, (1960), 12-14. 
[2] M. L. Buzano, Generalizzazione della diseguaglianza di Cauchy-Schwarz. (Italian), Rend. Sem. Mat. Univ. e Politech. Torino, 31, (1974), 405-409.

[3] S. S. Dragomir, Some refinements of Schwartz inequality, Simpozionul de Matematici şi Aplicaţii, Timişoara, Romania, 1-2 Noiembrie 1985, 13-16.

[4] S. S. Dragomir, Grüss inequality in inner product spaces, The Australian Math Soc. Gazette, 26, (1999), 66-70.

[5] S. S. Dragomir, A generalization of Grüss' inequality in inner product spaces and applications, J. Math. Anal. Appl., 237, (1999), 74-82.

[6] S. S. Dragomir, Some Grüss type inequalities in inner product spaces, J. Inequal. Pure \&3 Appl. Math., 4, (2003), Article 42. (Online http://jipam.vu.edu.au/article.php?sid=280).

[7] S. S. Dragomir, Reverses of Schwarz, triangle and Bessel inequalities in inner product spaces, J. Inequal. Pure \& Appl. Math., 5, (2004), Article 76. (Online : http://jipam.vu.edu.au/article.php?sid=432).

[8] S. S. Dragomir, New reverses of Schwarz, triangle and Bessel inequalities in inner product spaces, Austral. J. Math. Anal. \&3 Applics., 1, (2004), Article 1. (Online: http://ajmaa.org/cgi-bin/paper.pl?string=nrstbiips.tex).

[9] S. S. Dragomir, On Bessel and Grüss inequalities for orthornormal families in inner product spaces, Bull. Austral. Math. Soc., 69, (2004), 327-340.

[10] S. S. Dragomir, Advances in Inequalities of the Schwarz, Grüss and Bessel Type in Inner Product Spaces, Nova Science Publishers Inc, New York,x+249 p., 2005.

[11] S. S. Dragomir, Reverses of the Schwarz inequality in inner product spaces generalising a Klamkin-McLenaghan result, Bull. Austral. Math. Soc., 73, (2006), 69-78.

[12] S. S. Dragomir, Advances in Inequalities of the Schwarz, Triangle and Heisenberg Type in Inner Product Spaces., Nova Science Publishers, Inc., New York,xii+243 pp. ISBN: 978-1-59454-903-8; 1-59454-903-6 (Preprint http://rgmia.org/monographs/advancees2.htm), 2007.

[13] S. S. Dragomir, Some new Grüss' type inequalities for functions of selfadjoint operators in Hilbert spaces, Sarajevo J. Math., 6, (2010), 89107.

[14] S. S. Dragomir, Inequalities for the Čebyšev functional of two functions of selfadjoint operators in Hilbert spaces, RGMIA Res. Rep. Coll., 11(e), (2008), Art. 17.

[15] S. S. Dragomir, Some inequalities for the Čebyšev functional of two functions of selfadjoint operators in Hilbert spaces, RGMIA Res. Rep. Coll., 11(e), (2008), Art. 8.

[16] S. S. Dragomir, Inequalities for the Čebyšev functional of two functions of selfadjoint operators in Hilbert spaces, Aust. J. Math. Anal. \& Appl., 6, (2009), Article 7, pp. $1-58$.

[17] S. S. Dragomir, Some inequalities for power series of selfadjoint operators in Hilbert spaces via reverses of the Schwarz inequality, Integral Transforms Spec. Funct., 20, (2009), $757-767$. 
[18] S. S. Dragomir, Operator Inequalities of the Jensen, Čebyšev and Grüss Type, Springer Briefs in Mathematics. Springer, New York, xii+121 pp. ISBN: 978-1-46141520-6, 2012.

[19] S. S. Dragomir, Operator Inequalities of Ostrowski and Trapezoidal Type, Springer Briefs in Mathematics. Springer, New York, $\mathrm{x}+112$ pp. ISBN: 978-1-4614-1778-1, 2012.

[20] S. S. Dragomir, Inequalities for the Numerical Radius of Linear Operators in Hilbert Spaces, Springer Briefs in Mathematics. Springer, $\mathrm{x}+120$ pp. ISBN: 978-3-319-014470; 978-3-319-01448-7, 2013.

[21] S. S. Dragomir, M. V. Boldea, C. Buşe, and M. Megan, Norm inequalities of Čebyšev type for power series in Banach algebras, J. Inequal. Appl., 2014:294, (2014), 19 pp.

[22] S. S. Dragomir and B. Mond, On the superadditivity and monotonicity of Schwarz's inequality in inner product spaces, Contributions, Macedonian Acad. of Sci and Arts, 15, (1994), 5-22.

[23] S. S. Dragomir and B. Mond, Some inequalities for Fourier coefficients in inner product spaces, Periodica Math. Hungarica, 32, (1995), 167-172.

[24] S. S. Dragomir, J. Pečarić, and J. Sándor, The Chebyshev inequality in preHilbertian spaces. II., Proceedings of the Third Symposium of Mathematics and its Applications (Timişoara, 1989), (1990), 75-78.

[25] S. S. Dragomir and J. Sándor, The Chebyshev inequality in pre-Hilbertian spaces. I., Proceedings of the Second Symposium of Mathematics and its Applications (Timişoara, 1987), (1988.), 61-64.

[26] K. E. Gustafson and D. K. M. Rao, Numerical Range, Springer-Verlag, New York, Inc., 1997.

[27] S. Kurepa, Note on inequalities associated with Hermitian functionals, Glasnik Matematčki, 3, (1968), 196-205.

S. S. Dragomir ${ }^{1,2}$

${ }^{1}$ Mathematics, College of Engineering \& Science

Victoria University, PO Box 14428

Melbourne City, MC 8001, Australia.

E-mail: sever.dragomir@vu.edu.au

URL: http://rgmia.org/dragomir

${ }^{2}$ DST-NRF Centre of Excellence in the Mathematical and Statistical Sciences, School of Computer Science \& Applied Mathematics,

University of the Witwatersrand,

Private Bag 3, Johannesburg 2050, South Africa

Received: 4.11.2016

Accepted: 4.12 .2016 